\title{
Microscopic Analyses of 316 L Stainless Steel Powder from Additive Layer Manufacturing Process
}

H M Davies ${ }^{1}$, S Mehmood $^{2}$, A Khaliq $^{3}$, S A Ranjha ${ }^{4}$

${ }^{1}$ College of Engineering, Swansea University Bay Campus, Swansea SA1 8EN, UK

2 Northern Ireland Advanced Composites \& Engineering Centre, 5 Airport Road, Belfast BT3 9EF, UK

${ }^{3}$ Department of Materials Engineering (MTM), KU Leuven, 3001, Leuven, Belgium

${ }^{4}$ Nebraska Transportaion Center, University of Nebraska - Lincoln, 130, Prem S. Paul Research Center, United States

Metallic powders are used to manufacture complex parts using Additive Layer Manufacture (ALM) process in the aerospace, automotive and bio-medical sectors [1]. Although the technology has grown in recent years, it still faces many manufacturing and raw material related challenges. Typical manufacturing challenges include the lack of achieving close dimensional tolerances when compared to traditional machining [2] and a lower fatigue life due to the inherent internal porosity in parts manufactured via ALM [3]. Internationally, studies are ongoing in an attempt to resolve these challenges.

Producing metallic powders with suitable physical characteristics, e.g. particle size distribution, powder morphology and also the desired alloy chemistry for use in Additive Manufacturing processes, is a challenging task from both an economic and technical point of view. One of the solutions to this is to re-use the metallic powder which has been exposed to the laser heat inside the manufacturing chamber but not actually used within the manufactured build, subsequently termed "post-processing powder". Such powder needs to be analysed microscopically for its morphology and chemical composition before it substitutes virgin powder in ALM. In the present study $316 \mathrm{~L}$ Stainless Steel powder is analysed using Scanning Electron Microscopy with Energy Dispersive XRays (EDX) analyses. Two powder conditions are examined, namely,

- Virgin powder - as manufactured via Gas Atomisation in the "as received" condition from the supplier and has undergone no processing and,

- "Post processed powder" that has been exposed to the laser treatment during processing within the ALM chamber.

Virgin powder is spherical in shape and displays a smooth, surface (Figure 1a). However, as a result of the laser heat effect, post-processed powder shows noticeable changes to both the powder morphology and composition. Figure $1 \mathrm{~b}$ shows a typical powder agglomerate in which small powder particles are attached to a larger particle. Such agglomerates are unwanted as they can adversely affect the flowability and packing of powders during ALM manufacturing. These agglomerates must be dispersed to restore the powder flowability before such powder can be reused in the ALM process.

In addition, raised circular regions $(\sim 15 \mu \mathrm{m})$ were observed on the surface of the post-processed powder (Figure 2a). These raised regions, displayed chemical compositions different to that of the 316 L stainless steel master alloy. In particular these regions, as analysed via EDX analyses, showed higher Chromium levels but reduced Nickel and Iron levels (Figure 2b). Such localised variation in 
the chemical composition of the powder can introduce un-wanted characteristics, e.g. loss of corrosion protection, into the final build parts. A homogenisation heat-treatment is recommended as a trial to attain the chemical homogenisation inside the post-processed powder before re-reusing it in ALM processing or other applications.

\section{References:}

[1] Cherry J. A. et al, Int J Adv Manuf Technol 76 (2015), p.869.

[2] Townsend A. et al, Precision Engineering 46 (2016), p.34

[3] Chan K. S. et al, Metallurgical and Materials Transactions A 44 (2013), p.1010.

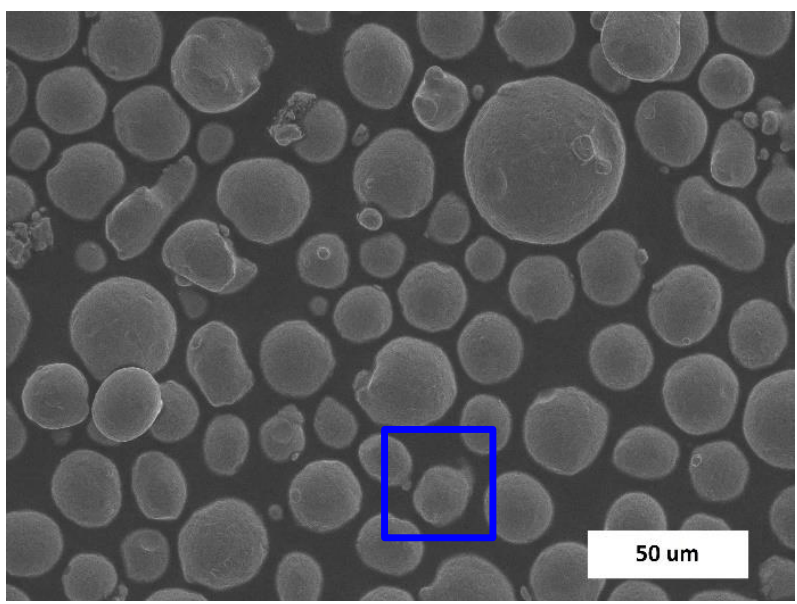

a) Virgin "As-received" 316 L Powder

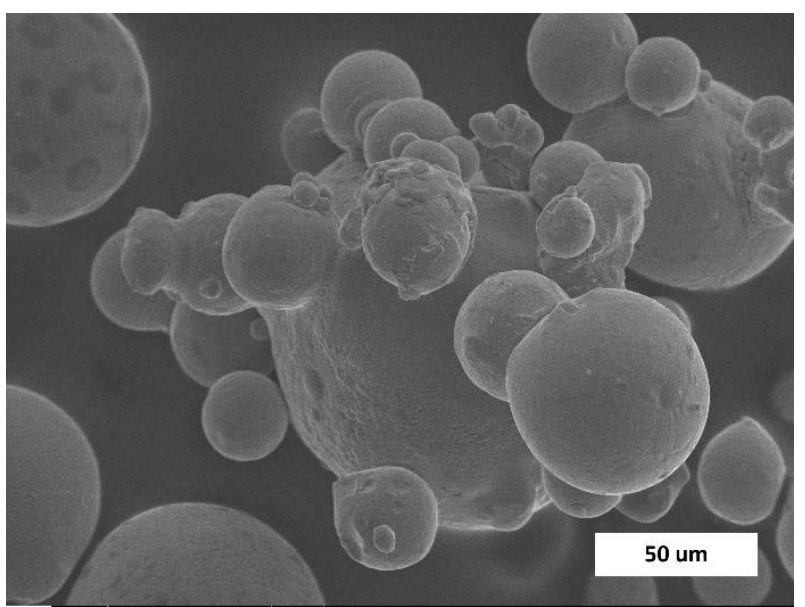

b) Used 316 L Powder

Figure 1. Microscopic analyses under SEM of ALM grade $316 \mathrm{~L}$ stainless steel powders highlighting surface features

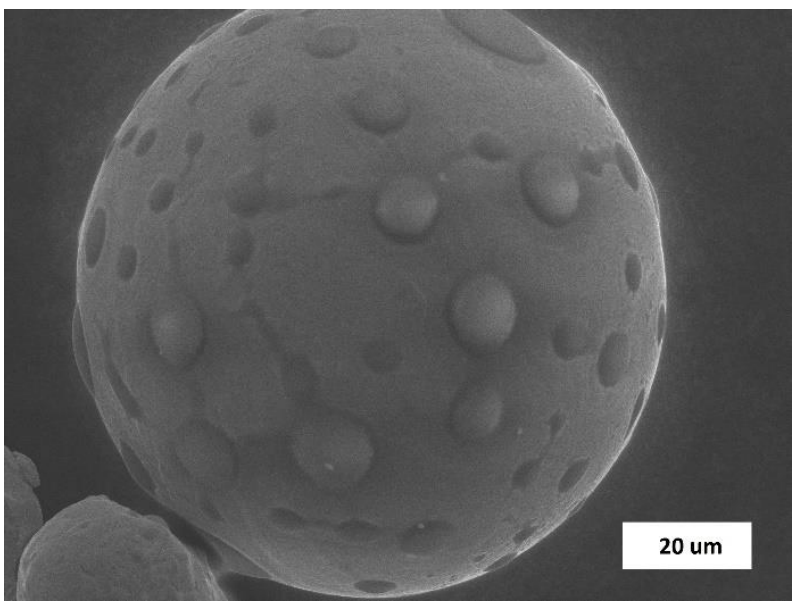

a) Used $316 \mathrm{~L}$ powder showing satellite formation

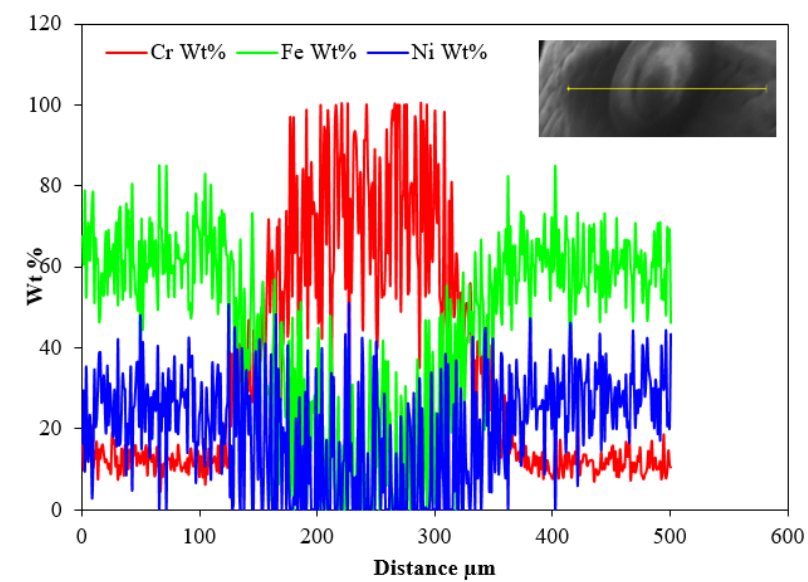

b) Line scan through region enclosed in blue box on Figure $2 \mathrm{a}$

Figure 2. Microscopic analyses under SEM/EDX of used $316 \mathrm{~L}$ powder highlighting depletion of metallic elements in formed satellites 